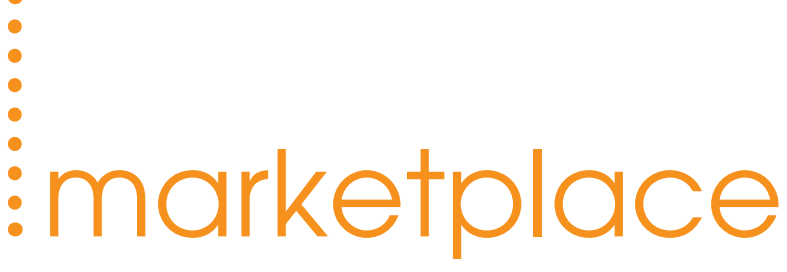

Marketplace is provided as a service to readers using text and images from the manufacturer, supplier or distributor and does not imply endorsement by Vital. Normal and prudent research should be exercised before purchase or use of any product mentioned.

\section{A natural alternative}

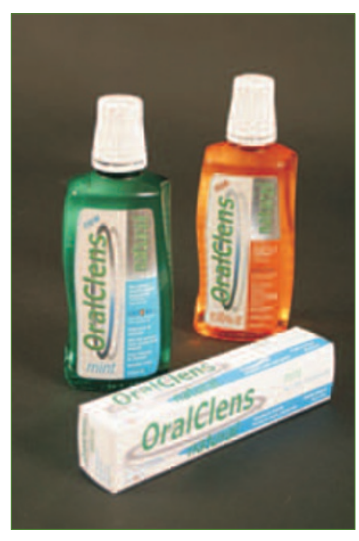

OralClens Natural from Oraldent is a new range of toothpaste and mouthwash, the active ingredients of which are 100\% natural. As a natural alternative to conventional, synthetic products it is kinder and gentler on the mouth and gums.

OralClens Natural toothpaste and mouthwash is a unique oral cleansing solution that contains a powerful anti-bacterial extracted from the pith of oranges, called Citroxx. The patented blend of non-toxic bioflavinoids and fruit acids contains no alcohol, no SLS, no synthetic anti-bacterials, and no synthetic sweeteners or flavourings. In addition the Citroxx natural antibacterial will protect against cavities and kill the germs that can cause bad breath.

Suitable for both adults and children, OralClens Natural will leave your patients' mouths feeling fresher and cleaner and is available in fresh mint flavour or tasty citrus. For a free sample, email Oraldent on sales@oraldent.co.uk.

\section{Sticky success}

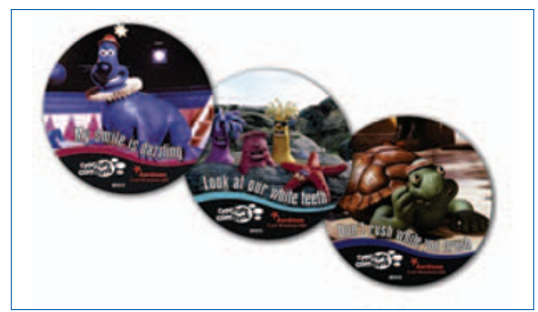

Sessions of York understand how difficult it is to motivate young children to clean their teeth. Already a supplier of 'dental motivators' to dentists across the UK, Sessions have recently added a new cast of characters to their range of stickers.

The new characters, from the stable of Creature Comforts, have trotted over to Sessions of York for a period of two years, to encourage young children to brush their teeth and maintain good dental hygiene habits.

Supplied on a reel, the labels are available to any dental practice supplier in the UK, for the purpose of encouraging the young not to 'rush while they brush', have dazzling and happy smiles, and educating them that, for example, ice cream is all right when teeth are brushed twice a day.

Characters such as Frank the Tortoise, Trixie and Captain Cuddlepuss are designed to motivate children to practise healthy dental habits in an entertaining way. For further information telephone 01904 659224, or visit www.sessionsofyork.co.uk.

\section{A faster recovery}

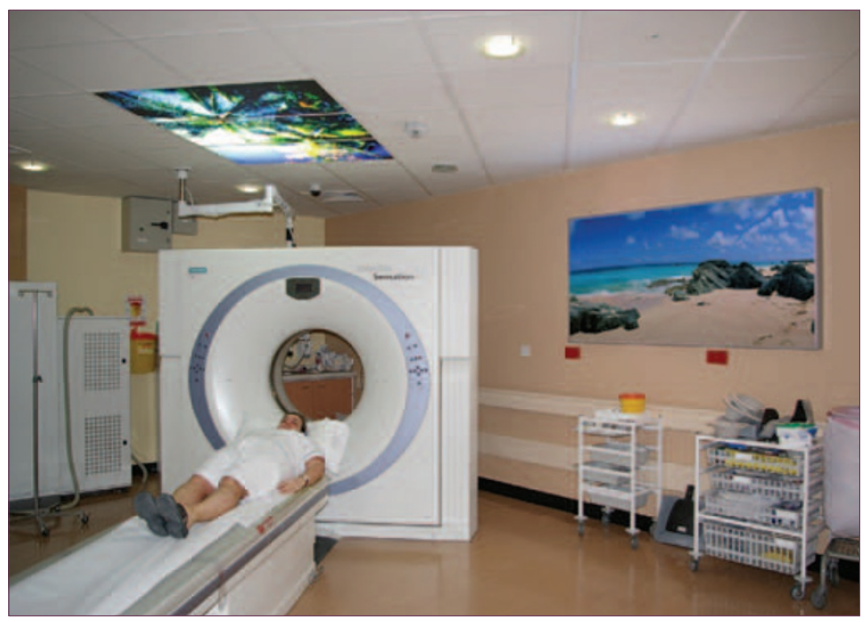

An increasing amount of scientific research suggests that images of nature during clinical procedures can dramatically improve patient stress levels, reduce systolic blood pressure, and minimise boredom - lessening the likelihood of complications and the need for expensive repeat diagnosis or treatment.

Visual Therapy, offered by diagnostic imaging specialists MAS Medical Limited, is a range of over 2,000 images by award winning nature photographer, Joey Fischer. In a healthcare setting such as a dental practice, images are used in both clinical and non-clinical areas to promote a healing environment.

Images are usually supplied as backlit transparencies and can be either wall or ceiling mounted, with wall mounted images supplied in custom made light boxes. Ceiling images are configured to fit within the ceiling grid or can be fitted in bespoke light boxes. The company offer advice with regards to size, position and image selection. For example, in cancer services, autumn scenes are not usually recommended because this season is often associated with an ending. For more information visit www.mas-medical.co.uk.

\section{Quick, powerful cleaning}

Specialist washer disinfector dryer manufacturer Dawmed International Ltd has created the Dawmed Clinic Washer Disinfector, which allows for quicker, more powerful cleaning of dental instruments.

The Clinic has the ability to process large and small loads including a combination of dental handpieces, and completes thermal disinfection of the internal lumens in a 15 minute cycle.

Once loaded, the Clinic's washing and thermal disinfection programme is simple to operate and fully automatic, leaving staff free to concentrate on other tasks. The Clinic's forced air function also allows for quicker cleaning, as dry instruments can be packaged immediately, or transferred directly to the steriliser. For more information visit www.dawmed-clinic.com or call 01709731356 